\title{
The Research of Environment Data Monitoring In Smart Home
}

\author{
Yuesheng Wang ${ }^{1, a}$, Yanfang Gan ${ }^{2, b}$ \\ ${ }^{1}$ School of Automation, Hangzhou Dianzi University, Hangzhou, 310018, China; \\ ${ }^{2}$ School of Automation, Hangzhou Dianzi University, Hangzhou, 310018, China; \\ awys@hdu.edu.cn, b1306080249@qq.com
}

Key words: Smart Home; TD-LTE; embedded processors; alarm in defensive area.

\begin{abstract}
As is known to all, home is an important part of the whole social system. With the great changes have taken place in the way of life and life concept, the requirements of the environment in Smart Home are becoming strict increasingly. So I design a system with sensors, the communication module of TD-LTE, the embedded processors, peripheral function module and so on. When the sensors are sensing the unreasonable situation, through the mobile network, the processor reflects to our mobile phones with SMS or making a phone call accompanied by sound and light alarm. As the same time, the system can be able to specify the number to call, hang up, answering the phone, text messaging and other basic function of communication. Using the system, the problems of poor security of traditional living environment such as gas leak or invasion of thief, can be deal with in time.
\end{abstract}

\section{Introduction}

Because of oversight, home environment where we have a reunite, a rest, is a fire-prone area. Such as gas leak, thieves burglary. So, with expect of a safe, healthy and comfortable home environment, the application of Smart Home was born.

This paper is under Smart Home's environmental monitoring and security module. Using the system, deals with the problems of poor security of traditional living environment such as gas leak or invasion of thief in time; helps upgrade the industrial structure and adjust economic strategic; has practical significance to green indoor living environment.

\section{The Overall System Design}

\subsection{System Works}

When the passive infrared sensor detects infrared signal comes from body radiation, when the smoke sensor senses a fire signal, when the carbon monoxide sensor senses the carbon monoxide concentration which exceeds a threshold value, the system convert those signals to weak electric signal, then the signal processing circuit deal with through filter, amplify, comparison, then export high level as the form of warning signals which to be send to embedded processor which export control signal to have a alarm with sound and light when meet the entry conditions. As the same time, through the mobile network of TD-LTE, the processor reflects to our mobile phones with SMS or making a phone call accompanied by sound and light alarm.

\subsection{System Structure}

System consists of three-layer structure: firstly, environmental information collections function: through the indoor passive infrared sensors, smoke sensors and carbon monoxide sensors, we can perceive the information of indoor air. Perception of information is the bottom and basic functions of the system. Secondly, environmental information transmissions function: mainly TD-LTE wireless communication module, which relies on the mature and reliable mobile network. Thirdly, information processing function: the processor of AT91SAM9G10 responsible for dealing with the information which pass over the environmental [4]. If the information exceeds the value set by user, it will text message to inform the user. If not, the information will be stored in the SQL database which be convenient for user to view the history state information. Figure 1 shows the overall 
system level.

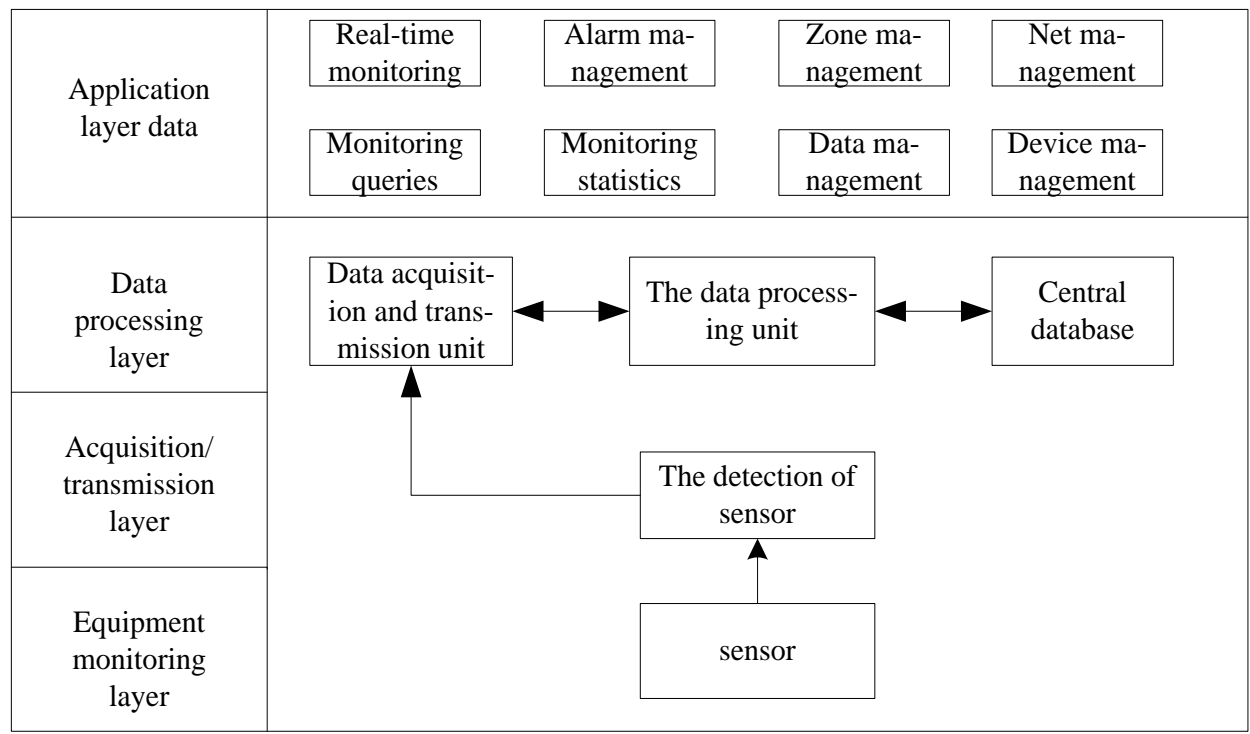

Figure 1 overall system hierarchy graph

\section{Hardware Design}

System consists of four major modules: the sensor of infrared ray, smoke, carbon monoxide, the embedded processor, the wireless communication module, relays. Figure 2 shows the hardware design. When the sensor detects the changing signals, the system converts those signals to weak electric signal which to be deal with by the signal processing circuit to achieve monitoring function.

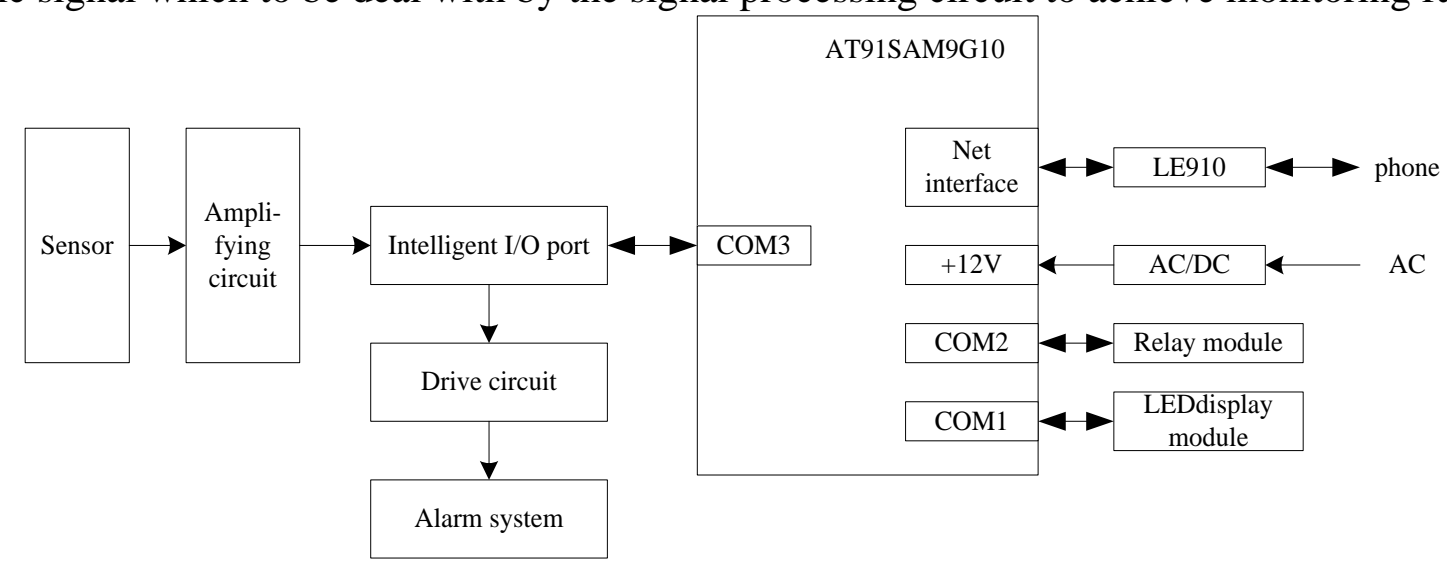

Figure 2 Hardware module diagram

\subsection{The Design of Wireless Communication Module}

LE910 is used in the wireless communication, the power range from 3.3 to $4.2 \mathrm{VDC}$, and the bands are LTE or UMTS. The module mainly responsible for: send and receive SMS, small quantities of remote data transmission, voice calls, real-time wireless data communication, and wireless online[5].

\subsection{The Design of Sensor}

First, the infrared sensor that is a kind of sensor to be detected infrared rays which release from human or animal. Firstly, it is passive, not actively emit infrared. When it is used indoor, there is no worried that outdoor sports can cause false alarm. Secondly, the probes of infrared sensor which model is HC-SR501, is sensitive to the infrared radiation which a wavelength about 10um.

Then, the carbon monoxide sensor which model is CO/CF-1000, when the carbon monoxide concentration reaches $100 \mathrm{ppm}$, the sensor output voltage may be able to reach $1 \times 10^{-4} \mathrm{v}$, then be send to amplifying circuit, the voltage can be 36 to 120 times.

Last, the smoke sensor which model is LH-91L is a type of switch sensor. Generally the output 
of the sensor is always off. But when it detects a certain concentration of smoke, the output of the sensor will be on with light and alarm signal[6].

\subsection{The Design of Processor}

AT91SAM9G10 is the processor we choose which the input voltage is $12 \mathrm{v}$, the output voltage is also $12 \mathrm{v}$. With two layers structure of floor and core board, the band is 200MHZ.We use ethernet1 to configure device parameters and ethernet 2 for data transmission and device parameters. Through a USB HOST, we can collect data with the 2.0 protocol. And a USB DEVICE write ARM MPU program. The input of 8-channel analog interface signals is $5 \mathrm{v}$ or $20 \mathrm{~mA}$. The 8-channel RS232 are link to intelligent instruments[7].

\section{The Design of System Software}

\subsection{Design of System Software Architecture}

When the sensor senses an abnormal condition in defense area, the system can be directly reflected to the phone which be specified. At the same time, the system can hang up the phone, answer the phone, text messaging and other basic communication functions. Therefore, in case of emergency, the user can turn on the phone to monitor and megaphone. Figure 3 shows the main program flow chart.

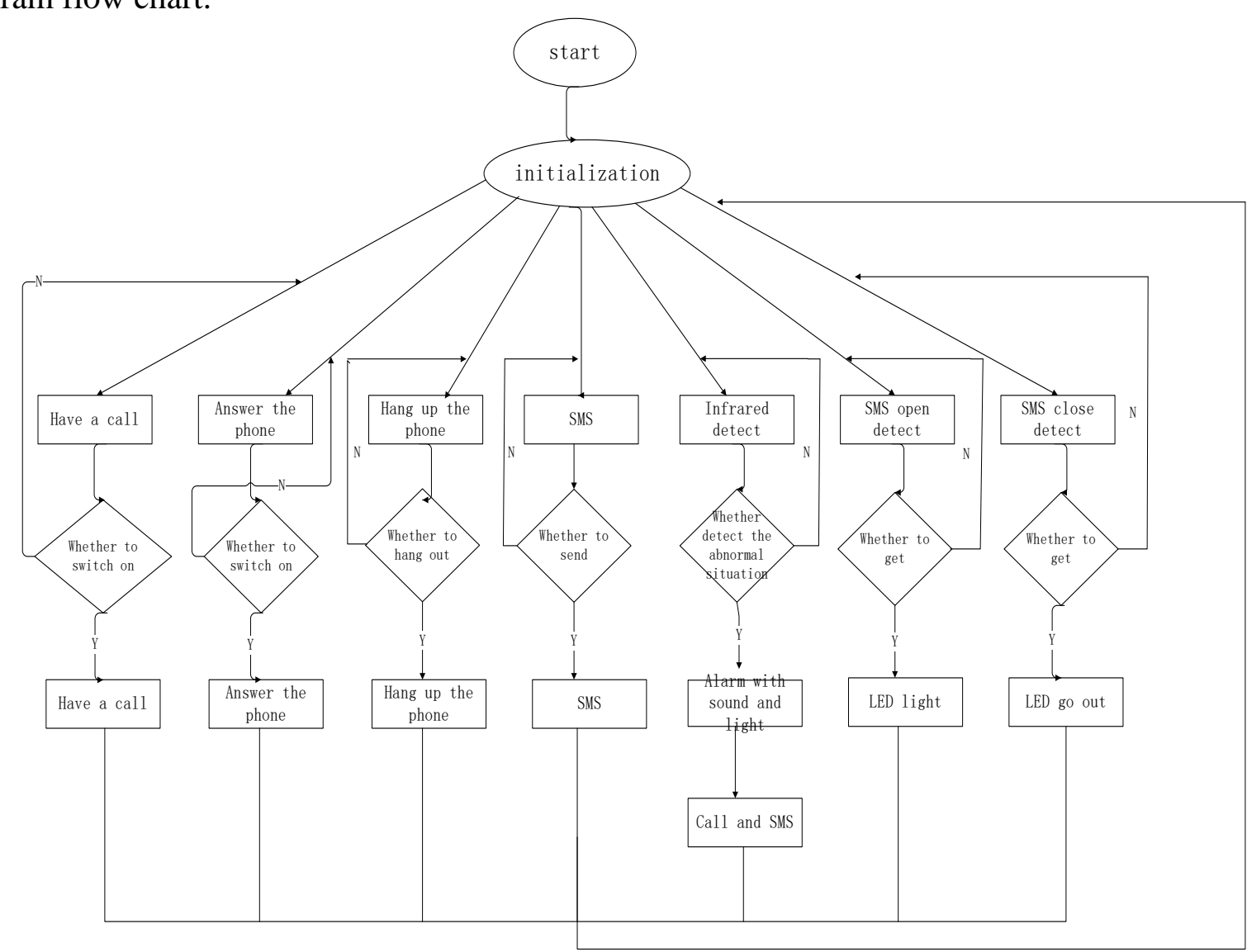

Figure 3 The main program software diagram

\subsection{The Design of Sensor Software}

When the sensor detects an abnormal condition, sensor module VO-side output high level, then it is necessary to down the corresponding port of processor by program for conveniently detecting whether an interrupt occurs. This design uses INTO, when accept the interrupt signal, processor will have interrupt processing. Figure 4 shows the program flow chart. 


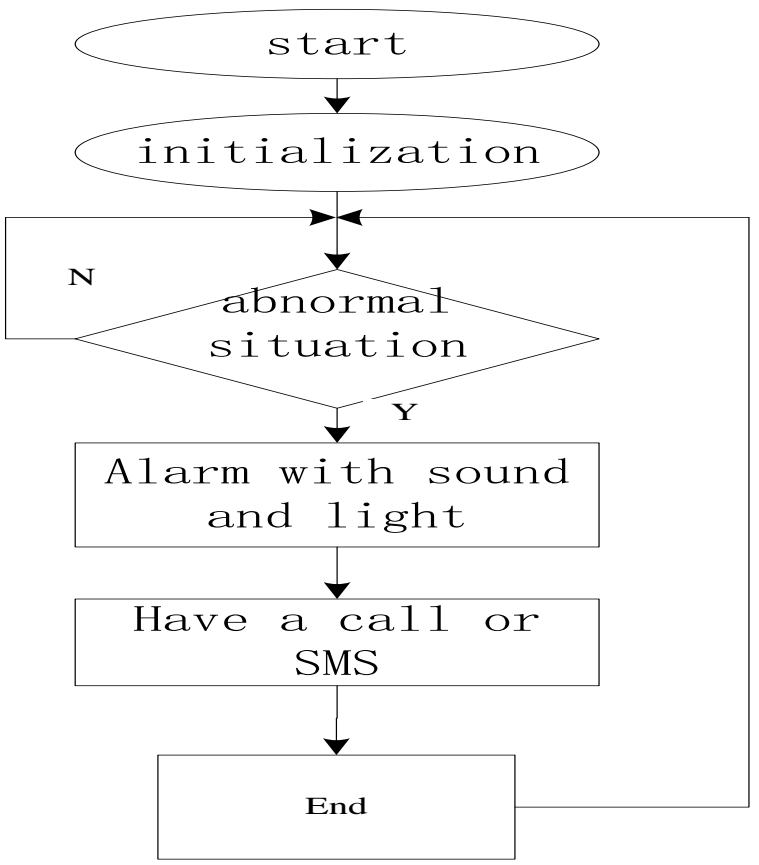

Figure 4 The sensor software diagram

\section{Conclusions}

With the most reliable and most mature TD-LTE mobile network, this system directly reflects the situation in the protection zones to the mobile phone for users doing the corresponding processing. At the same time, the system can hang up the phone, answer the phone, text messaging and other basic communication functions. Therefore, in case of emergency, the user can turn on the phone to monitor and megaphone.

So, the research of this system helps to solve the problem of traditional living environment which to be poor in safety, such as timely deal with gas leak, thieves burglary, fire and other issues; And helps to have a green indoor life which makes people's life more efficient and convenient, and lets the family numbers to avoid useless calories in daily activities.

\section{References}

[1] Liao Xichun. The Remote Intelligent Smart Home Monitoring System Based on Bluetooth [J].Wireless communication technology, 2010.

[2] Han Jianghong, Zhang Jianjun, Zhang Li. Smart Home system and technology [M]. Hefei: the Press of Hefei University of Technology, 2009.

[3] Jiang Ting, Zhao Chenlin. Smart Home Technology and Application [M].Beijing: the Press of Beijing University of Posts and Telecommunications, 2013.

[4] Zhou Hong, Zhang Liming. Smart Home control system [M]. Beijing: China Electric Power Press, 2014.

[5] You Liang.IEE802.15.4 Standard and Applications [J].Electronic Design and Application, 2010.

[6] Wang Quanping, Wang Li. Zigbee Technology and Application [J].Technology of Telecommunications, 2009.

[7] Liu Song, Zhao Zhong, Sun Xuelei.Smart Home Remote Monitoring System Based on GSM[J].Electronic Measurement Technology, 2009. 